\title{
CATEGORIZACIÓN DE SERVICIOS DE ENFERMERÍA. PROPUESTA PARA ASEGURAR UNA ATENCIÓN DE CALIDAD EN TIEMPOS DE ESCASEZ DE ENFERMERAS ${ }^{1}$
}

\section{NURSING SERVICES CLASSIFICATION: PROPOSAL TO ENSURE QUALITY CARE IN TIMES OF NURSING SHORTAGES}

\author{
Paulina Milos ${ }^{*}$ \\ ANA IsABEL LARRAIN ${ }^{*}$ \\ MARTA SiMONETTI $^{* * *}$
}

\begin{abstract}
RESUMEN
Numerosos estudios han comunicado sobre la crisis mundial de escasez de enfermeras. Chile no se encuentra ajeno a este fenómeno. Ante esta contingencia se propone adoptar el modelo de división del trabajo propuesto por Benoit-Smullyan, generando el situs de enfermería, la categorización de servicios sustentada en criterios de delegación/encargo a las demás ocupaciones del situs y proyectar la Gestión del Cuidado como centro de producción de acciones de enfermería. El desarrollo del situs de enfermería, la categorización de servicios y la delegación/encargo son consideradas herramientas efectivas que evitan la sustitución de funciones, aumentan la producción de acciones de enfermería seguras, de calidad e inspiradas en los valores de la profesión.
\end{abstract}

Palabras claves: Dotación de enfermeras, servicios de enfermería, gestión del cuidado.

\begin{abstract}
Numerous studies have reported on the shortage of nurses global crisis. Chile is not beyond this phenomenon. In view of this situation, we propose the adoption of the model of division of work described by Benoit-Smullyan, generating the nursing situs, the categorization of services based on certain delegation/commissioning criteria to the other occupations within the situs, and to foster Nursing Care Management as the center of nursing actions' production. The development of the nursing situs, the categorization of services and the delegation/commissioning are considered effective tools to prevent the substitution of functions and to enhance the production of safe, quality nursing work, inspired in the values of the profession.
\end{abstract}

Keywords: Nursing staff, nursing services, nursing care management.

Fecha recepción: 09/03/09 Fecha aceptación: 24/03/09

\footnotetext{
${ }^{1}$ Resumen presentado en el XVI Congreso Nacional de Enfermeras. La Serena, Chile, octubre 2007. Se publicó un extracto como Carta al Editor, en la Revista de Calidad Asistencial, 2008; 23: 286.

* Enfermera; Licenciada en Derecho, Abogado. Email: paulinamilosh@uandes.cl, Dirección : San Carlos de Apoquindo 2200, Las Condes, Santiago, Chile.

** Enfermera-matrona; directora Escuela de Enfermería Universidad de los Andes. Santiago, Chile. Email: alarrain@uandes.cl ${ }_{* * *}$ Enfermera-matrona; directora de Estudios Escuela de Enfermería. Universidad de los Andes. Santiago, Chile. Email: msimonetti@uandes.cl
} 


\section{INTRODUCCIÓN}

Múltiples estudios han abordado la crisis mundial de escasez de enfermeras. Recientemente el Informe Salud 2006 de la Organización Mundial de la Salud (OMS) da cuenta de que hay aproximadamente cuatro enfermeras por cada médico en Canadá y EEUU, mientras que en Chile, menos de una por cada médico(1). Recientemente se ha informado un promedio de 780,4 enfermeras registradas por cada 100.000 habitantes, señalando que cifras de 626,1 por 100 mil personas se encuentran muy por debajo de las necesidades de la población.(2) La media europea, para el año 2000, se encontraba en 755 enfermeras por 100.000 habitantes(3). En Chile no se maneja este tipo de estadísticas, pudiéndose sobre la base del número de médicos(4) y su relación con el número de enfermeras que da cuenta la OMS estimar una cifra de aproximadamente 600 enfermeras por 100.000 habitantes. En EEUU, en el año 2006 existían 126.000 puestos vacantes para enfermeras, señalándose que para el año 2020 habrá 400.000 enfermeras menos de las que se necesitan en el país.(5) Una fuente autorizada en nuestro país ha dado cuenta de un déficit de un $47 \%$ de enfermeras en el ámbito hospitalario; este déficit aumenta a un $60 \%$ en los centros de atención primaria.(6)

Por otra parte, cada vez más se reportan los beneficios de los servicios proporcionados por enfermeras sobre la disminución de la morbi-mortalidad y sobre la satisfacción usuaria. Tourangeau (2007) concluye que los hospitales con alta proporción de enfermeras con formación superior reducen significativamente la tasa de mortalidad.(7) Asimismo, ha quedado demostrado el impacto de una buena relación entre el número de enfermeras y el número de pacientes en términos de resultados: menor mortalidad, menor índice de infecciones nosocomiales, úlceras por presión y complicaciones postoperatorias, estadía hospitalaria más corta, mejor calidad de la atención(8), mayor seguridad del paciente(9) y mayor satisfacción usuaria(10).

La escasez de enfermeras pone en riesgo una atención de enfermería integral, segura y de calidad. En efecto, se observa con mayor frecuencia que profesionales de otras disciplinas, y también auxiliares y técnicos, asumen funciones de la enfermera que importan un criterio profesional. El problema se torna crítico si se consideran las condiciones epidemiológicas actuales que generan mayores necesidades de cuidado, el envejecimiento profesional y el compromiso con la calidad en salud.(11) Todo esto hará que la demanda de enfermeras vaya en aumento.

El término escasez indefectiblemente se relaciona con el de dotación, entendida ésta como "el número y la clase de las personas necesarias para dispensar cuidados al paciente o cliente"(12). Entonces no sólo es importante el número de enfermeras sino también las competencias al interior de los equipos de enfermería. La operatividad depende del número, adecuado equilibrio de competencias y funciones que cada uno de sus miembros pueda aportar.

A modo de solución se han estudiado cargas de trabajo, categorización de pacientes, combinación de capacidades, sustitución de funciones y una serie de otras iniciativas e instrumentos, los que en su mayoría apuntan a las variables tiempo y costo. Estas medidas coadyuvan a la solución del problema más, por sí solas, no lo solucionan.

La falta de adecuación a la realidad generada por la escasez de enfermeras tiene tanto efectos inmediatos como a mediano y largo plazo: baja en la producción, calidad y seguridad de los servicios de enfermería, impacto sobre la eficacia de los equipos de salud y de la red asistencial $(1,11)$ y aumento de costos de la atención de salud. A esto se puede sumar una distorsión del modelo de enfermería: indicaciones que importan un criterio profesional radicadas en personas sin formación; socialización inadecuada de la profesión, y prestación de servicios de enfermería no ba- 
sados en la evidencia científica.

Conforme a la función social de la enfermera, el ethos profesional y su rol jurídico, se propone buscar alternativas de solución de modo de satisfacer las necesidades de cuidado de la población, preservando la humanización, calidad y seguridad de la atención. En el ámbito de la salud, se ha señalado que una estrategia eficaz dirigida a la fuerza laboral debe centrarse en tres objetivos fundamentales: 1) mejorar la contratación, 2) ayudar al personal existente a potenciar su desempeño y 3) ralentizar el ritmo de abandono de la fuerza laboral(1).La propuesta que se presenta se enmarca en el segundo de estos objetivos y apunta a hacer un uso más eficaz de las enfermeras existentes(13) y a mejorar la productividad de las acciones de enfermería(1) con sensibilidad a las necesidades de las personas, en forma justa y eficaz, y con los recursos de que se dispone. Al efecto se propone la conformación del "situs de enfermería", categorización de servicios de la enfermera y delegación/ encargo de determinados servicios a las demás ocupaciones del situs. Por último, se trata la responsabilidad por los actos y acciones generadas bajo el modelo que se propone.

\section{CONFORMACIÓN DEL SITUS DE ENFERMERÍA}

La función social de la enfermera: En el año 1997, la sociedad chilena reconoce que el problema social que resuelven las enfermeras es la satisfacción de la necesidad de cuidados. Conforme a ello la Ley No 19.536 define su rol social, el que comprende tres grandes funciones: la gestión del cuidado, la ejecución de acciones derivadas del diagnóstico y tratamiento médico y el deber de velar por la mejor administración de recursos de asistencia para el paciente(14). El análisis que se hará a continuación se centrará principalmente en la gestión del cuidado y en el deber de velar por la mejor administración de recursos de asistencia de los pacientes.

La gestión del cuidado: se entiende por gestión del cuidado la aplicación de un juicio profesional en la planificación, organización, motivación y control de la provisión de cuidados(15). El cuidado, si bien es de la esencia del quehacer de la enfermera, en la práctica puede ser brindado por un técnico de nivel superior, por otro profesional o por un auxiliar de enfermería(16). La gestión del cuidado, en cambio, es lo que la profesión ofrece de forma única a la sociedad. Ésta representa el conocimiento abstracto de la profesión y su capacidad para resolver problemas de forma discrecional. La gestión del cuidado interviene las variables críticas de la atención de enfermería: acceso, oportunidad, humanización, seguridad, calidad y reducción de costos.

La gestión del cuidado es intransferible en cuanto que requiere de un criterio profesional que, desde su planificación hasta su ejecución, responda a estándares éticos, jurídicos y científico-técnicos, que sólo se alcanzan con una formación superior impregnada de la tradición y evolución histórica de la enfermería. A través del ejercicio de la gestión del cuidado (y el cuidado) la enfermera cumple su función social, lo que significa que la profesión resuelve un problema social determinado, como no lo podría hacer ninguna otra profesión.

El cuidado de enfermería: es el conjunto de acciones sistematizadas, organizadas, medibles, documentables y evaluables, basadas en modelos y teorías de enfermería y/o en la evidencia científica, realizadas con el fin de garantizar que una persona, con problemas reales o potenciales de salud, reciba una atención de enfermería segura, eficiente y eficaz, cuya finalidad sea lograr su máximo bienestar; y el amparo y protección de sus valores y derechos, amenazados por su estado de carencia o enfermedad(17). El cuidado de la salud de las personas es el objetivo principal de la profesión.

La gestión del cuidado y el cuidado conforman una unidad representativa del quehacer de la enfermera, donde la gestión 
sólo será delegable a otra enfermera, en cambio el cuidado podrá ser encargado, bajo indicación y supervigilancia de una enfermera, en otro profesional o en el personal auxiliar.

El deber de velar por la mejor administración de recursos de asistencia para el paciente: significa que la enfermera asume la posición de garante de la asistencia del paciente en un sentido integral. Se trata de un mandato social que, si bien es de connotación administrativa, trasunta un alto contenido ético. $\mathrm{Al}$ encomendar el legislador a las enfermeras el deber de velar por la mejor administración de los recursos de asistencia para el paciente, éstas son incorporadas como garantes y agentes éticos de los derechos e intereses de los usuarios. La posición de garante, desde el punto de vista jurídico, es una obligación. Su incumplimiento podría ser sancionado por acción u omisión. En cumplimiento de esta función, a la enfermera le cabe la responsabilidad de distribuir el trabajo (delegación/ encargo), establecer criterios de asignación y límites de los servicios de enfermería y educar en el uso racional de los recursos, de modo de incrementar su eficacia y efectividad. De igual manera, es responsable de asesorar el diseño y ejecución de los procesos de reclutamiento, selección, orientación, supervisión, evaluación, asignación, suspensión y despido del personal de enfermería. Asimismo, a fin de garantizar la calidad, continuidad y oportunidad en el cuidado le corresponderá participar en los procesos de adquisición y suministro de equipos e insumos.

El situs de enfermería: el modelo de distribución social del trabajo propuesto por Benoit-Smullyan(18) distingue dos dimensiones: una horizontal o situs, referida a la función básica que cumple una profesión, y una vertical referida a la distribución jerárquica de las ocupaciones que conforman el situs. La ocupación que, por su condición de poseer la totalidad o casi la totalidad de los conocimientos necesarios para desempeñar las diferentes funciones del situs, se ubica en el vértice de éste(19).
La profesión de enfermera genera diversas ocupaciones u oficios. Sin embargo, el situs de enfermería, siendo una realidad, no ha sido desarrollado. Al situs en su conjunto le cabe la responsabilidad de satisfacer las necesidades de cuidado que demanda la población. La enfermera tendrá que asegurar la eficiencia del situs por medio de la complementación de las actividades que se desarrollan a su interior(20). Para ello deberá coordinar y controlar las actividades de todas las ocupaciones que lo componen; estructurar y controlar la enseñanza que deben recibir los integrantes de las diferentes ocupaciones; elaborar las normas éticas y técnicas que guíen el desempeño de las diversas ocupaciones; construir equipos y crear una cultura de colaboración entre los integrantes del situs(20). En la consecución de la eficiencia del situs deberá generar un entorno laboral favorable, forjando una cultura de sensibilidad por la necesidad de cuidado de las personas enfermas, promoviendo el aprendizaje permanente, gestionando los equipos e instaurando sistemas de responsabilidad(1).

Delegación / encargo: los avances tecnológicos y las condiciones actuales de formación de los integrantes de las ocupaciones auxiliares permiten la transferencia de acciones o tareas que no necesariamente debe realizar el profesional titular. La delegación es una transferencia de tareas, funciones, atribuciones y autoridad, que se realiza entre una persona que ocupa un cargo superior a un inferior jerárquico para que opere en un campo limitado y acotado, normalmente con un objetivo específico $(20,21)$. La delegación es dar, de una persona a otra, la jurisdicción que se tiene por su oficio para que: a) haga unas tareas, caso en que se denominará encargo y b) la represente, caso que se tratará de una delegación propiamente tal(22). El delegante conserva siempre la responsabilidad final por el resultado. El delegado tiene la obligación de rendición de cuentas al superior de las tareas que han sido delegadas.

La delegación / encargo en el plano admi- 
nistrativo ha demostrado, entre otros, que ahorra tiempo, incrementa la participación, potencia capacidades gerenciales, incrementa la motivación e innovación, libera la presión del trabajo inmediato y reduce costos de la empresa ya que el directivo puede dedicarse a otras funciones. Desde el punto de vista social, la delegación / encargo permite que se cubran las necesidades de cuidados de enfermería de la población bajo criterios científico-técnicos y estándares éticos profesionales. A la vez, hace posible el desarrollo del situs de enfermería con sus consecuencias benéficas. Permite el desarrollo de la profesión de enfermera en áreas que hoy demandan los nuevos modelos de atención. No obstante, se han observado algunos riesgos: la delegación de tareas sin justificación, la retrodelegación, y la delegación permanente ${ }^{20}$. Por esto, se hace necesario que las enfermeras adquieran las competencias para delegar y que conozcan los requisitos y las responsabilidades que la delegación involucra(23).

\section{RESPONSABILIDAD AL INTERIOR DEL SITUS}

La responsabilidad penal de los ocupantes del situs es personalísima, es decir, cada cual responde de sus propios actos. En el ámbito civil, en cambio, la enfermera será responsable en forma directa por haber elegido mal a quien encomendar una tarea (culpa in eligendo) o por vigilancia insuficiente en el cumplimiento del encargo (culpa in vigilando). Incluso, podría llegar a ser responsable, indirectamente, por el hecho de los dependientes (culpa in operando). No obstante, en este último caso se podrá eximir de responsabilidad "si con la autoridad y el cuidado que su respectiva calidad les confiere y prescribe, no hubiere podido impedir el hecho" (Art. 2330, inciso final; Código Civil chileno).

En el caso del trabajo en equipo, la doctrina y la jurisprudencia penal han desarrollado y acogido el "principio de la confianza". Merced a este principio, cada uno debe esperar que los demás actúen como es debido. La aplicación de este principio no es ilimitada, teniendo su excepción relativa en casos que se requiera que el superior deba entregar instrucciones precisas, informar, supervisar y dirigir a sus subalternos. Caso en que éste es responsable de la corrección de todos los actos que de él dependen. Sin embargo, si el subalterno actúa sin indicación ni supervisión o era imposible de prever un determinado comportamiento, las consecuencias recaen directamente sobre su persona(24). En todo caso, la aplicación de esta excepción será directamente proporcional al deber de cuidado del comportamiento de otras personas(25). La división del trabajo debe tener como soporte la confianza; pudiendo en virtud de este principio quien encarga llegar a eximirse de toda responsabilidad, aun en caso de un hecho culposo del dependiente que cause daño. En relación a la división vertical del trabajo, se ha señalado que "el que ocupa la posición superior puede confiar, en principio, en que sus instrucciones serán seguidas y el que ocupa una posición inferior, en que las instrucciones recibidas serán correctas". "En la división de trabajo vertical el principio de confianza juega en toda su intensidad"(26). De esta forma, queda claramente delimitada la responsabilidad, el campo y parámetros dentro de los cuales se puede imputar a los ocupantes del situs un hecho que cause daño.

\section{LA CATEGORIZACIÓN DE LOS SERVICIOS DE LA ENFERMERA}

Categorizar, para los fines de esta propuesta, significa clasificar los servicios según la factibilidad de ser transferidos. La categorización se basa en la evaluación objetiva de las funciones y actividades de la enfermera conforme a la legislación vigente, a los conceptos de delegación/encargo y a la capacidad resolutiva 
frente a determinados problemas.

Provisionalmente proponemos clasificar los servicios según las siguientes categorías: 1 . Servicios productivos, núcleo de producción de actos y acciones de enfermería, generadores de productos finales. Estos servicios son los que legitiman la existencia de las enfermeras ya que recogen sus funciones principales. 2. Servicios asistenciales, los que comprenden los cuidados directos, cuidados indirectos, bajo indicación y supervisión, la educación del paciente y familia e interconsultas de enfermería, entre otros. 3. Productos intermedios, son aquellos que se realizan en apoyo y soporte de productos finales(27). Surgen del cumplimiento de otros servicios, conexos a la profesión, pero que se cumplen con entidad propia, por ejemplo una intervención iniciada por parte de un médico u otro profesional sanitario, pero llevado a cabo por una enfermera. También se encuentran dentro de esta categoría los actos de naturaleza médica, que en casos de urgencia, a falta de médico, son realizados por la enfermera. 4 . Servicios de coordinación y enlace (directivos), se trata de servicios que la enfermera no podrá encargar. En su ejercicio le cabrá integrar comisiones, comités, mesas intersectoriales o interinstitucionales, redes asistenciales, otros. 5. Servicios de apoyo a la tecno-estructura: son aquellos que posibilitan los servicios productivos de las áreas financiera, recursos humanos, asesoría jurídica, gestión de reclamos, admisión, recaudación, informática, etcétera(28).

Mediante la categorización se pretende ayudar a la organización y productividad de la atención de enfermería y a clarificar y explicitar las relaciones dentro del situs, lo que por extensión permitirá evaluar la capacidad de la dotación de enfermeras.

\section{CONSIDERACIONES FINALES}

Ante la escasez de enfermeras, el desafío es la búsqueda de soluciones oportunas, justas, centradas en el bienestar y seguridad del paciente. Al efecto se propone: 1) Asumir "el situs de enfermería" como modelo de división del trabajo y como estrategia para lograr universalidad en el reconocimiento y control de las acciones de enfermería. 2) Regular las formalidades de la delegación/ encargo dentro del situs. 3) Categorizar, provisoriamente, los servicios de la enfermera en servicios: productivos, asistenciales, productos intermedios, de coordinación y enlace, de apoyo a la tecno-estructura. 4) Estudiar otras categorías y modalidades de categorización de servicios. 5) Promover la reglamentación correspondiente.

Frente al problema de escasez de enfermeras parece recomendable que éstas se concentren en la gestión del cuidado en cuanto núcleo de producción de acciones de enfermería. Las enfermeras, al resguardar y multiplicar la calidad en los cuidados, responderán a su función social.

\section{REFERENCIAS}

1. Organización Mundial de la Salud. Colaboremos por la salud. Informe sobre la salud en el mundo. [en línea] 2006 [fecha de acceso 15 de agosto de 2007]. Disponible en: http://www.who.int/whr/2006/es

2. Giovine P. Preocupa escasez de enfermeras. [en línea] 2004. [fecha de acceso 9 de septiembre de 2007]. Disponible en: http:// www.univision.com/content/content. jhtml?cid $=502108$

3. Organización Mundial de la Salud. Health for all database. [en línea] 2000. [fecha de acceso 15 de agosto de 2007]. Disponible en : http://www.who.org

4. Bastías G, Marshall G, Zúniga D, Mena B. Número de médicos en Chile: estimaciones, proyecciones y comparación internacional. Rev med Chile 2000; 128(10):1167-1176.

5. Consejo Internacional de Enfermeras. Problemas mundiales de la oferta y la demanda 
de enfermeras. Noticias $S E W N^{\circ} 1$. [en línea] 2003 [fecha de acceso 9 de septiembre de 2007]. Disponible en: http://www.icn.ch/ sewjan-mar03sp.htm

6. Olivares R. Fuga de enfermeras hacia el sector privado provoca grave déficit en hospitales públicos. El Mercurio. 2009, febrero 2. Disponible en: http://diario.elmercurio. $\mathrm{cl} /$ detalle/index.asp? $\mathrm{id}=\{\mathrm{c} 438 \mathrm{~b} 2 \mathrm{~b} 6-62 \mathrm{~b} 7-$ 4592-919b-814ffd6b5fd4

7. Tourangeau AE, Doran DM, McGillis L, O’Brien-Pallas LL, Pringle D, Tu JV et al. Impact of hospital nursing care on 30-day mortality for acute medical patients. Journal of Advanced Nursing 2007; 57(1):3244.

8. U.S. Department of Health \& Human Services. Agency for Healthcare Research and Quality. Los cuidados supervisados por enfermeras mejoran el funcionamiento vital de pacientes con insuficiencia cardiaca que residen en comunidades minoritarias. Press Release. [en línea] 2006 [fecha de acceso 9 de septiembre de 2007]. Disponible en: http://www.ahrq.gov/news/press/pr2006/ spnursepr.htm

9. Mesa L. La enfermera es la primera línea de defensa en la seguridad del paciente. Evidentia 2007; 4 (18).

10. Joint Commission on Accreditation of Healthcare Organizations. Health care at the crossroads: Strategies for addressing the evolving nursing crisis. [en línea] 2002. [fecha de acceso 15 de agosto de 2007]. Disponible en: http://www.jointcommission. org/NR/rdonlyres/5C138711-ED76-4D6F909F-B06E0309F36D/0/health_care_at_ the_crossroads.pdf

11. Consejo Internacional de Enfermeras. La escasez mundial de enfermeras: Sectores de intervención prioritaria. Informe CIE / FIFN. [en línea] 2006. [fecha de acceso 5 de septiembre de 2007]. Disponible en: http:// www.icn.ch/global/shortagesp.pdf

12.McGillis L, Editor. Quality work environments for nurse and patient safety, Canadá. Jones and Bartlett Publications; 2005;
9-37.

13. O'Brien-Pallas L, Thomson D, Alksnis C, Luba M, Pagniello A, Ray K et al. Stepping to success and sustainability: An analysis of Ontario's nursing workforce. [en línea] 2003 [fecha de acceso 20 de septiembre de 2007] Disponible en: http://www.fhs.mcmaster.ca/nru/documents/Stepping\%20 to $\% 20$ Success $\% 20$ and $\% 20$ Sustainability\%202003.pdf

14. Código Sanitario. Decreto con Fuerza de Ley No 725. Actualizado a julio de 2000. Libro V, Artículo 113, inciso cuarto. Disponible en: www.bibliotecadelcongreso. cl/.../pub_leyes_mas_soli/admin/ver_archivo_codigos.php?id_codigo $=6 \&$ file $=1$

15. Ministerio de Salud. Norma General Administrativa No 19. Gestión del Cuidado de Enfermería Para la Atención Cerrada, Exenta No 1127 [en línea] 2007 [fecha de acceso 29 de diciembre de 2007]. Disponible en: http://www.colegiodeenfermeras. $\mathrm{cl} /$ datos/ftp/norma.doc

16. Rodríguez JA, Guillén MF. Organizaciones y profesiones en la sociedad contemporánea. REIS, 1992; 59: 9-17.

17. Fundación Víctor Grífols i Lucas. La gestión del cuidado en enfermería. Cuadernos No12. Barcelona: Fundación Víctor Grífols i Lucas; 2005.

18. Morris RT, Murphy RJ. The Situs Dimension in Occupational Structure. American Sociological Review, 1959; 24(2): 231-239; en Rodríguez JA, Guillén MF. Op. cit.

19. Gyarmati G, et al. Las Profesiones Dilemas del Conocimiento y del Poder. Santiago, Chile: Ediciones Universidad Católica de Chile; 1984: p. 65.

20. Musso F. Delegación y retrodelegación de la función directiva. Documentos de Apoyo Docente. Santiago, Chile: Universidad de Chile, Instituto de Asuntos Públicos; 2005: 14-25.

21. Indalecia P. Cambio Organizacional en el campo de la enfermería. Monografía. [en línea] 2000. [fecha de acceso 5 de septiembre de 2007]. Disponible en: http://www. 
monografias.com/trabajos39/cambioenfermeria/cambio-enfermeria.shtml

22. Castro M. Conceptos y recomendaciones para la delegación. Santiago, Chile: Hkmk Consultores; 2005:1-4.

23. Consejo Internacional de Enfermeras. Desarrollo de la reglamentación, las funciones y la competencia. Informe CIE / FIFN. [en línea] 2006. [fecha de acceso 5 de septiembre de 2007]. Disponible en: http://www. icn.ch/global/Issue1RegulationSP.pdf

24.López MJ. Tratado de responsabilidad médica: responsabilidad civil, penal y hospitalaria. Buenos Aires: Legis Editores S.A; 2007.

25.Galán JC. Responsabilidad civil médica.
Madrid: Civitas; 2005.

26. Barreiro AJ. Nuevos aspectos de la imprudencia jurídico-penal en la actividad médica: la culpa en el equipo médicoquirúrgico. Responsabilidad del Personal Sanitario; 1995.

27.Instituto Superior de Investigación para la Calidad Sanitaria ISICS. Proyecto NIPE Normalización de las Intervenciones para la Práctica Enfermera, 2002; anexo 1:189192.

28. Villalbi JR, Guix J, Plasencia A, Armengou JM, Llebaría X, Torralba L. La cartera de servicios en una organización de salud pública [Adaptación]. Gac Sanit 2003; 17(3):231-237. 